\title{
Swimming improves memory and antioxidant defense in an animal model of duchenne muscular dystrophy
}

\section{Priscila Mantovani Nocetti}

UNISUL Campus da Grande Florianopolis: Universidade do Sul de Santa Catarina - Campus da Grande Florianopolis

Adriano Alberti ( $\square$ adrianoalberti90@hotmail.com )

Universidade do Sul de Santa Catarina - Campus da Grande Florianopolis https://orcid.org/0000-00030313-0380

\section{Viviane Freiberger}

UNISUL Campus da Grande Florianopolis: Universidade do Sul de Santa Catarina - Campus da Grande Florianopolis

\section{Letícia Ventura}

UNISUL Campus da Grande Florianopolis: Universidade do Sul de Santa Catarina - Campus da Grande Florianopolis

\section{Leoberto Ricardo Grigollo}

UNISUL Campus da Grande Florianopolis: Universidade do Sul de Santa Catarina - Campus da Grande Florianopolis

\section{Cristina Salar Andreau}

CEU Cardenal Herrera University: CEU Universidad Cardenal Herrera

\section{Rudy José Nodari Junior}

UNOESC Curso de Medicina: Universidade do Oeste de Santa Catarina Curso de Medicina

\section{Daniel Fernandes Martins}

UNISUL Campus da Grande Florianopolis: Universidade do Sul de Santa Catarina - Campus da Grande Florianopolis

\section{Clarissa Martinelli Comim}

UNISUL Campus da Grande Florianopolis: Universidade do Sul de Santa Catarina - Campus da Grande Florianopolis

\section{Research Article}

Keywords: Duchenne muscular dystrophy. Animal. Swimming. Oxidative stress. Mice

Posted Date: May 7th, 2021 
DOl: https://doi.org/10.21203/rs.3.rs-496821/v1

License: (c) (1) This work is licensed under a Creative Commons Attribution 4.0 International License. Read Full License

Version of Record: A version of this preprint was published at Molecular Neurobiology on July 10th, 2021. See the published version at https://doi.org/10.1007/s12035-021-02482-y. 


\section{Abstract}

Duchenne muscular dystrophy (DMD) is a genetic disease, which is associated to progressive skeletal muscle degeneration. In humans, DMD has early onset, causes developmental delays, and is a devastating disease that drastically diminishes the quality of life of the young individuals affected. The objective of this study was to evaluate the effects of a swimming protocol on memory and oxidative stress in an animal model of Duchenne muscular dystrophy. Male $m d x$ and wild type mice within 28 days were used in this study. The animals were trained in a stepped swimming protocol for four consecutive weeks. It was verified that swimming was able to reduce significantly the levels of lipid peroxidation and protein carbonilation in gastrocnemius and hippocampus and striatum in exercised animals. Swimming has also prevented lipid peroxidation in diaphragm. Besides, this swimming protocol was able to increase free thiols in gastrocnemius, diaphragm and in all central nervous system structures. These results showed that a protocol of swimming as an aerobic exercise of low intensity, for four weeks, prevented aversive memory and habituation in $m d x$ mice.

\section{Introduction}

Duchenne muscular dystrophy is a well-described clinical disease; it is a condition caused by an amendment to the $X$ chromosome, inherited as a recessive trait, and mainly affects males [1, 2]. Affected animals such as $m d x$ mice have the same mutation as that in humans. They are of limited use as they do not develop early dilated cardiomyopathy as seen in humans; however, they are the best option for experimentation $[1,3]$.

Patients diagnosed with DMD require a multidisciplinary treatment approach, which must be careful and always focus on the well-being of the patient. Therefore, the role of the physical therapy is important for a successful treatment, once it has achieved good outcomes in the short term, such as maintaining the autonomy of these individuals $[4,5]$. Physical exercises are also used to treat DMD [6, 7]. Studies have been using physical exercises in individuals with DMD to decrease muscle deterioration, muscular contraction, and bone fractures and increase the duration of functional independence [8]. Low-intensity exercise is known to decrease certain parameters associated with muscular degeneration in animal models of progressive muscular dystrophies [2].

Swimming is an extremely popular aerobic exercise modality. Aerobic physical exercise in water is a viable alternative to land-based exercise because water, through its physical properties, such as thrust, hydrostatic pressure, and viscosity, increases the well-being and quality of life [9]. Swimming, as well as hiking and running, has many health benefits when compared to a sedentary lifestyle [9]. Swimming is generally indicated for avoiding impact and facilitating compliance because of a better adaptation to an environment with heated water [9]. The literature also shows that swimming has beneficial effects on the patient's cognition, by modulating memory and learning processes, and on oxidative stress, by increasing antioxidant defenses [9]. Therefore, this study aims to evaluate the effects of swimming on memory and oxidative stress in an animal model of DMD. 


\section{Materials And Methods}

\subsection{Animals}

Male $m d x$ mice and wild-type C57BL/6 mice, kindly provided by University of São Paulo (USP), Brazil, were used in this study. The animals were 28 days old, and they weighed 18-23 g. The animals were kept in the Vivarium of the Experimental Neuroscience Laboratory (Lanex), in Unisul, Palhoça, Santa Catarina, Brazil. Each box contained five animals, in a 12-h light-dark cycle ( $6 \mathrm{am}$ to $6 \mathrm{pm})$, with food and water provided ad libitum. The temperature of the environment was maintained at $22 \pm 2^{\circ} \mathrm{C}$. The study was conducted at Lanex and at the Laboratory of Biochemistry and Molecular Biology of Unisul, Palhoça, Santa Catarina, Brazil, during 2016 and 2017. Approval for conducting this study was obtained from the Animal Use Ethics Committee (CEUA) of Unisul under the number 16.003.4.01.IV.

\subsection{Sample calculation}

To calculate the number of animals required for each experimental group, we have to take: $n=[(z a / 2+z \beta)$ $2 . s 2] /(-x-\mu)^{2}$. Where:

- $z a / 2=z$ value on normal curve according to a value;

- $z \beta=$ beta error;

- $s 2$ = variance;

- $(X-\mu)^{2}=$ estimated maximum difference between the sample mean $(X)$ and the true population mean $(\mu)$. It is the error margin or maximum estimation error.

Therefore, the following values are assigned to calculate the number of animals:

1. The alpha value will be set at 0.05 . Hence, the $z a / 2$ value, based on the table of $z$-values for twotailed distribution, is 1.96 .

2. The beta value will be set at 0.20 . Hence, the $z$ beta value, based on the table of $z$-values (singletailed distribution), is 0.84 .

3. The value of the standard deviation (s) is on average $30 \%$ of the means' value (based on experimental data from our laboratory).

The difference between the groups' means is at least $40 \%$ (based on experimental data from our laboratory). Biological experiments have an embedded error of about $10-15 \%$ (resulting from individual variations, surgical procedure errors, dosage errors, etc.).

Assigning the values to the above formula, we have:

$\mathrm{n}=[(1,96+0,84) 2.302] /(0,4-0,1) 2=7,84$ animals

It is therefore understood that a minimum of eight animals should be used in each experimental group to ensure that the experiments' conclusions are valid, within an acceptable risk of not observing differences 
where they exist and not seeing differences where they do not exist. This study used 10 animals per group to guarantee data safety and reliability.

\subsection{Experimental design}

As illustrated in Fig. 1. The animals were divided into the following four groups, each one consisting of eight animals: (1) non-exercised wild-type, (2) exercised wild-type, (3) non-exercised $m d x$, and (4) exercised $m d x$. Groups 2 and 4 were subjected to the low intensity aerobic exercise protocol of the swimming type for 4 weeks. After 24 hours of the last day of training, the tests for assessing aversive memory and habituation were conducted. Afterwards, the animals were sedated and submitted to the assisted painless death procedure, and the following structures were removed for determining lipid peroxidation, protein carbonylation, and thiol grouping: the gastrocnemius and quadriceps muscles, diaphragm, prefrontal cortex, cerebellum, hippocampus, striatum, and cerebral cortex.

\subsection{Aerobic exercise protocol of swimming type}

The groups of exercised animals were submitted to a protocol of swimming aerobic exercise in a plastic container adapted for this purpose $(170 \times 100 \mathrm{~mm})$, with 35 liters of water at 28 to $30^{\circ} \mathrm{C}$ degrees, divided into eight lanes. The protocol consisted on four consecutive weeks of exercise, four times a week, with daily 15-minute sessions in the first week, 20 minutes in the second week, 30 minutes in the third and fourth weeks (adapted from [10]). It was used $1 \mathrm{ml}$ of baby's shampoo all over the container to decrease the surface tension of the water (adapted from [11]). After the protocol, the animals were gently dried. The groups of non-exercised animals did not perform any type of physical exercise, remaining in their housing boxes during the entire study period.

The determination of the exercise intensity was performed on the fourth week of protocol in the wt e $m d x$ animals. Blood samples were collected before the test, on the $10^{\text {th }}$ and $30^{\text {th }}$ minutes of exercise for subsequent analysis of lactate concentration. The criterion for considering intensity was the increase in concentration of no more than $1 \mathrm{mmol} / \mathrm{L}$ between the $10^{\text {th }}$ and $30^{\text {th }}$ minutes of physical exercise. The blood collection was performed in an alcohol $(70 \%)$ sanitized place. After this procedure, the distal portion of the animal's tail was slightly sectioned with surgical scissors and $25 \mu$ of a drop of blood was inserted in the lactate collection tape, and through a portable lactimeter, the blood lactate level was measured. Before each test, the equipment was calibrated according to the manufacturer's instructions [12].

\subsection{Aversive memory test}

This test consists of an acrylic box whose floor is formed by parallel metal bars. A platform $7 \mathrm{~cm}$ wide and $2.5 \mathrm{~cm}$ long is placed near the left wall of the appliance. At the training session, the animals are placed on the platform, and the time taken by the animal to go down the platform with the four legs is measured, in seconds. This time is called latency. Immediately after going down the platform, the animal receives a $0.2 \mathrm{~mA}$ shock for two seconds. In the test session, the animal is placed on the platform again, 
and the time it takes to go down (latency) is measured; however, the shock is not administered. The test is also finalized if the animal does not go down within three minutes [13].

\subsection{Habituation test}

This test was performed in a $40 \times 60 \mathrm{~cm}$ open field, delimited by four $50 \mathrm{~cm}$ high walls - three made of wood and one of transparent glass. The open field floor is divided into 16 equal squares, marked by black lines. In the training session, the animals were carefully placed in the square of the rear left corner of the appliance, from which they freely explored the environment for five minutes. Immediately afterward, the animals returned to the housing box. The test session was held 24 hours after the training, in which the training procedure is repeated. The number of four-legged crossings (crossings: motor activity) through the black lines and the number of times the animals rested on their back legs (rearings: exploratory activity) were evaluated in both sessions [14].

\subsection{Oxidative stress measures}

\subsubsection{Measurement of thiobarbituric acid reactive substances (TBARS)}

This method is used to evaluate the oxidation state of hydroperoxides in biological systems. Damage to membrane lipids is determined by the formation of lipoxidation by-products (such as MDA or malondialdehyde), which are reactive substances to thiobarbituric acid heating, formed during peroxidation in membrane systems. MDA reacts with thiobarbituric acid (TBA), generating a pinkishcolored product, read in a $535 \mathrm{~nm}$ microplate reader. The technique consists of the following: first of all, the dilution value was calculated so that in the TBARS reaction tube there are $100 \mu \mathrm{l}$ of tissue protein in $500 \mu \mathrm{l}$ of BHT buffer. Afterwards, $500 \mu \mathrm{l}$ of the $0.67 \%$ TBA solution were added. The tubes were placed in a dry bath at $96^{\circ} \mathrm{C}$ degrees for 30 minutes. To stop the reaction, the samples were placed on ice for five minutes. Finally, $200 \mu \mathrm{l}$ of the reaction were placed in 96 -well microplates and read in the microplate reader at $535 \mathrm{~nm}$.

\subsubsection{Measurement of oxidative damage in proteins}

This method was used for protein oxidation dosing. It is based on the principle that several ROS attack protein residues, such as amino acids to produce products with the carbonyl group, which can be measured though the reaction with 2,4-dinitrophenylhydrazine. The carbonyl content is determined by a microplate reader at $370 \mathrm{~nm}$, as described by Levine et al. [15]. First, the tissue was homogenized in $1 \mathrm{ml}$ BHT buffer.

The samples were centrifuged for $15 \mathrm{~min}$ at $4{ }^{\circ} \mathrm{C}$ at $14,000 \mathrm{rpm} .200 \mu \mathrm{l}$ of the sample were separated for the blank and $200 \mu \mathrm{l}$ for the test. $100 \mu \mathrm{l}$ of $20 \%$ trichloroacetic acid (TCA) were placed in all eppendorfs. They were centrifuged for five minutes at 14,000 rpm. The supernatant was discarded. The pellet was redissolved in $100 \mu$ of 0.2 molar $\mathrm{NaOH} .100 \mu$ dinitrophenylhydrazine 2.4 (DNTP) were placed in the sample and were left to rest for one hour. $100 \mu \mathrm{l} 20 \%$ TCA were placed on all eppendorfs, which were centrifuged for three minutes. The supernatant was discarded. The pellet was washed three times with 
$500 \mu$ l ethanol and ethyl acetate (1: 1). For each wash, it was centrifuged for three min at 14,000 rpm and the supernatant discarded. After discarding the last wash, $1 \mathrm{ml}$ of $3 \%$ sodium hydroxide $(\mathrm{NaOH})$ was placed on all eppendorfs. The samples were taken to water bath at $60^{\circ} \mathrm{C}$ for 30 minutes and read in the microplate reader at $370 \mathrm{~nm}$.

\subsection{Thiol groups}

Sulfhydryl radicals represent all groups of thiols found in proteins such as albumin and low molecular weight compounds, such as glutathione. These groups can be oxidized when oxidative stress is high. The determination of total sulfhydryl groups, protein-linked sulfhydryl groups, sulfhydryl groups in low molecular weight compounds (free sulfhydryl) can be performed by using Ellman's reagent (2,2-dinitro5,5-dithiobenzoic acid - DTNB). The thiol groups react with DTNB forming a light-absorbing complex at $412 \mathrm{~nm}$. The technique consists of adding 10\% TCA to the same sample volume (1: 1 dilution). White was prepared, containing $100 \mu \mathrm{l} \mathrm{TCA}$ by adding $100 \mu \mathrm{l} \mathrm{PBS}$. It was centrifuged for 15 minutes at 3,000 rpm (temperature $4{ }^{\circ} \mathrm{C}$ degrees); the supernatant was collected, and $30 \mu$ ITNB $(1.7 \mathrm{mM})$ and $300 \mu \mathrm{l}$ hydrochloric acid (TRIS-HCl) were added to $75 \mu \mathrm{l}$ of this supernatant. It was left to react for 30 minutes and transferred to a 96 -well plate. The samples were read in a $412 \mathrm{~nm}$ microplate reader.

\subsection{Protein Dosages}

The proteins were determined by the BCA method and the bovine serum albumin was used as standard. The method is based on the reaction of the copper with the proteins in basic medium. The samples were analyzed by a plate reader at $562 \mathrm{~nm}$.

\section{Statistical Analysis}

The data were entered into a database, developed in electronic media, in IBM SPSS Statistics 24.0 software (@copyright IBM corporation and its licensors 1989, 2016). The Shapiro-Wilk normality test was applied to verify the behavior of the data. The data referring to the open field habituation test were expressed in average and standard deviation because they are parametric data. For differences between groups, the two-way ANOVA test with post hoc Bonferroni was used. For differences between training and testing in the same group, the student's t test was used for paired samples. The data of the inhibitory elusive test were expressed in median and interquartile range because they are non-parametric data. Wilcoxon test was used for analysis between training and test in the same group. The data from the biochemical tests were expressed as mean and standard deviation because they were parametric data. The two-way ANOVA test was used with post hoc Bonferroni for analysis between groups. Data were considered statistically significant when $p<0.05$.

\section{Results}

\subsection{Lactate measurement}


Figure 2 shows the results obtained after the lactate measurement made in the blood taken from the animals during the performance of the swimming protocol.

Figure 2 shows that in both $m d x$ and wt animals submitted to the swimming protocol, there were no changes in blood lactate above $1 \mathrm{mmol} / \mathrm{L}$ between the 10th and 30th minute of physical exercise in relation to the rest values (the average lactate values were $2,31 \mathrm{nmol} / \mathrm{l} / \mathrm{I}$ for wt animals and $2.21 \mathrm{for} m d x$ animals), in other words, they remained below the lactate threshold). Therefore, the swimming protocol used in this study can be considered of moderate intensity.

\subsection{Learning and memory evaluation}

Figure 3 shows the results obtained after the application of the swimming protocol on aversive memory (Fig. 3A) and habituation (Fig. 3B). In Fig. 3A, the results of the habituation memory evaluation, through the open field test, can be observed. It can be remarked that there was no difference in the number of crossings and rearings ( $p>0.05)$, between groups, during the training phase, demonstrating that there was no difference in locomotive activity between the groups. The animals wt (wild) non-exercised and exercised demonstrated significant changes, between training and testing, both in the number of crossings and the number of rearings $(p<0.05)$; in other words, there was no impairment of the evaluated memory. The group of $m d x$ (DMD) non-exercised animals did not show significant changes between training and testing in the number of crossings and rearings $(p>0.95)$, evidencing a memory impairment. In contrast, the group of $m d x$ animals that were submitted to the protocol showed a decrease in the number of crossings and rearings between training and testing $(p<0.05)$, suggesting a possible prevention to the impairment of aversive memory in $m d x$ mice.

The results of the aversive memory assessment through the inhibitory avoidance test are shown in Fig. 3B. In the group of non-exercised and exercised wt animals, there was a statistically significant difference in latency time between training and testing, showing no impairment in the aversive memory $(p<0.05)$. In the non-exercised $m d x$ group of animals, there was no statistically significant difference between training and testing, evidencing an impairment of aversive memory $(p>0.05)$. The $m d x$ animals submitted to the experimental protocol showed a statistically significant difference between training and testing, in other words, there was no impairment of aversive memory in these animals $(p<0.05)$.

\subsection{Oxidative stress assessment in the gastrocnemius muscle}

Figure 4 shows the results of using a swimming protocol on lipid peroxidation (Fig. 4A), protein carbonylation (Fig. 4B), and free thiols (Fig. 4C) in the gastrocnemius muscle.

Figure 4A shows the result of evaluation of lipid peroxidation in gastrocnemius. It was observed that the non-exercised $m d x$ animals presented significantly elevated levels of lipid peroxidation in gastrocnemius, when compared to untrained wild animals $(p<0.05)$. The $m d x$ animals submitted to the experimental protocol presented a significant reduction of these levels, when compared to the non-exercised $m d x$ animals $(p<0.05)$, evidencing that the experimental protocol used in this study has protected against the 
increase in lipid peroxidation in gastrocnemius in $m d x$ animals. It can be observed that the non-exercised $m d x$ animals showed a significant increase in protein carbonylation in gastrocnemius when compared to the group of non-exercised wild animals $(p<0.05)$. After the experimental protocol, the $m d x$ animals presented significantly lower protein carbonylation levels when compared to the non-exercised $m d x$ animals $(p<0.05)$, demonstrating that the swimming protocol, for four weeks, was able to prevent the carbonylation increase in proteins, observed in the gastrocnemius muscle of $m d x$ animals (Fig. 4B). The quantification of free thiols in gastrocnemius is shown in Fig. 4C. It can be observed that there was a significant increase in the number of free thiols in gastrocnemius of wild animals group submitted to the experimental protocol, when compared to the non-exercised wild animals $(p<0.05)$. There was also a significant decrease in the non-exercised $m d x$ animals when compared to the non-exercised wild animals $(p<0.05)$. When the $m d x$ animals were submitted to the experimental protocol, there was an increase in the number of free thiols, when compared to the $m d x$ non-exercised animals.

\subsection{Oxidative stress assessment on the diaphragm muscle}

Figure 5 shows the results of using a swimming protocol on lipid peroxidation (Fig. 5A), protein carbonylation (Fig. 5B), and free thiols (Fig. 5C) in the diaphragm muscle. It is observed that the nonexercised $m d x$ animals showed a significant increase in diaphragm lipid peroxidation when compared to the group of non-exercised wild animals $(p<0.05)$. After the experimental protocol, the $m d x$ animals showed significantly lower lipid peroxidation levels when compared to the non-exercised $m d x$ animals ( $p$ $<0.05)$, demonstrating that the swimming protocol, for four weeks, was able to prevent the increase in lipid peroxidation observed in the diaphragm muscle of $m d x$ animals (Fig. 5A). Figure 5B shows the result of the assessment of protein carbonylation in diaphragm. It was observed that the non-exercised $m d x$ animals have presented significantly higher levels of protein carbonylation in diaphragm when compared to the non-exercised wild animals $(p<0.05)$. However, the $m d x$ animals that were submitted to the experimental protocol did not present a significant reduction of these levels when compared to the nonexercised $m d x$ animals, evidencing that the experimental protocol used in this study did not protect against the increase in protein carbonylation in diaphragm in $m d x$ animals. The quantitation of the diaphragm free thiols is shown in Fig. 5C. It can be observed that there was a significant increase in the number of free thiols in diaphragm of the group of wild animals submitted to the experimental protocol, when compared to the non-exercised wild animals $(p<0.05)$. There was also a significant decrease in non-exercised $m d x$ animals when compared to non-exercised wild animals $(p<0.05)$. When the $m d x$ animals were submitted to the experimental protocol, there was an increase in the number of free thiols when compared to the non-exercised $m d x$ animals, showing that swimming was able to protect this change.

\subsection{Oxidative stress assessment in central nervous system structures}

Figure 6 shows the results of using a swimming protocol on lipid peroxidation in the prefrontal cortex (Fig. 6A), hippocampus (Fig. 6B), striatum (Fig. 6C), and cortex (Fig. 6D). 
It is observed that the non-exercised $m d x$ animals showed a significant increase on lipid peroxidation in hippocampus and striatum when compared to the group of non-exercised wild animals $(p<0.05)$. After the experimental protocol the $m d x$ animals showed significantly lower lipid peroxidation levels in the hippocampus and striatum when compared to the untrained $m d x$ animals $(p<0.05)$, demonstrating that the swimming protocol, for four weeks, was able to prevent the increase in the lipid peroxidation observed in the hippocampus and striatum structures of $m d x$ animals (Figs. $6 \mathrm{~B}$ and $6 \mathrm{C}$ ). Figures $6 \mathrm{~A}$ and $6 \mathrm{D}$ show that in the prefrontal cortex and cortex structures there were no significant changes between the groups analyzed. Figure 7 shows the results of using a swimming protocol on protein carbonylation in the prefrontal cortex (Fig. 7A), hippocampus (Fig. 7B), striatum (Fig. 7C) and cortex (Fig. 7D).

It can be observed that the non-exercised $m d x$ animals showed a significant increase in protein carbonylation in prefrontal cortex, hippocampus and striatum when compared to the group of nonexercised wild animals $(p<0.05)$. After the experimental protocol, the $m d x$ animals showed significantly lower protein carbonylation levels in the prefrontal cortex when compared to the untrained $m d x$ animals $(p<0.05)$, demonstrating that the swimming protocol, for four weeks, was able to prevent the increase in protein carbonylation, observed only in the prefrontal cortex structure of $m d x$ animals (Fig. 7A).

Figures 7B and 7C show that the swimming protocol, for four weeks, was not able to prevent the increase in protein carbonation in the hippocampus and striatum. Figure 7D shows that in the cortex structure there was no significant change between the analyzed groups.

Figure 8 shows the results of using a swimming protocol on free thiols in the prefrontal cortex (Fig. 8A), hippocampus (Fig. 8B), striatum (Fig. 8C) and cortex (Fig. 8D).

It can be observed that the non-exercised $m d x$ animals showed a significant decrease in free thiols in prefrontal cortex, hippocampus, striatum and cortex when compared to the group of non-exercised wild animals $(p<0.05)$. After the experimental protocol, the $m d x$ animals showed significantly higher free thiol levels in prefrontal cortex, hippocampus, striatum and cortex when compared to non-trained $m d x$ animals $(p<0.05)$, demonstrating that the swimming protocol, for four weeks, was able to increase the antioxidant defenses in all central nervous system (CNS) structures of $m d x$ animals (Figs. 8A, 8B, 8C and 8D).

\section{Discussion}

The effects of swimming on memory and oxidative stress on skeletal muscle and encephalic tissue were evaluated in this study using a DMD animal model.

For this purpose, a moderate-intensity swimming protocol was implemented, performed for four consecutive weeks, four times a week. The results showed that swimming prevented the impairment of aversive memory and habituation in $m d x$ mice. Parallel to this effect, by analyzing the parameters associated with oxidative damage, it was also observed an increase in protein carbonylation in the prefrontal cortex, hippocampus, striatum, diaphragm, and gastrocnemius and an increase in lipid peroxidation in the hippocampus, striatum, diaphragm, and gastrocnemius muscle, concomitant with a decrease in the free thiol levels in non-exercised $m d x$ animals, indicating oxidative stress. Interestingly, 
low-intensity swimming was able to prevent oxidative stress in the gastrocnemius and hippocampus and striatum structures of these animals. This exercise protocol also increased the free thiol levels in the gastrocnemius, diaphragm, and structures of the CNS analyzed in this study.

In this study, swimming (aerobic exercise) was considered of moderate intensity, according to lactate measurements made in the animals, and for that $1 \mathrm{~mL}$ of shampoo was used in the whole container adapted for swimming. On exercise intensity, besides the classification based on VO2max measurement and maximum heart rate, which classifies the exercise as mild/low, moderate or high intensity [16], there is also the model proposed by Gaesser and Poole [17] that indicates three domains in relation to effort intensity: moderate, heavy and severe. The moderate domain comprises all the effort intensities that can be performed without modifying blood lactate in relation to resting values, i.e., below the lactate threshold (LL). The heavy domain starts from the lowest effort intensity where lactate rises, and has, as upper limit, the intensity corresponding to an average of $4 \mathrm{MM}$ of lactate. In the severe domain, there is no stable phase of blood lactate; it rises during all the effort time until the individual is exhausted [17].

DMD is characterized by an absence of dystrophin protein in skeletal muscle [6]. However, the literature also states that dystrophin is absent in encephalic tissue, and this change is associated with other changes such as oxidative stress [18]. The absence of dystrophin and the oxidative stress in the CNS makes cognitive impairment part of the pathophysiology of this disease. In this study, it was evinced that the swimming protocol was able to protect the impairment of aversive memory and habituation in the $m d x$ mice submitted to the protocol.

The protocol of the present study was started with 28-day-old animals and completed at 56 days old. At this age, these animals would be expected to present memory and learning impairments, which did not occur in animals submitted to swimming. Thus, it can be stated that the exercise prevented memory and learning deficits. Although there are no studies relating swimming directly to the prevention of memory and learning impairment related to $\mathrm{DMD}$, there are reports of benefits of swimming practice in cognitive aging [19].

Another study, also with middle-aged animals, showed benefits of swimming, when combined with dietary supplementation, in object recognition memory tests, demonstrating short- and long-term memory improvement [20].

Besides cognitive impairment, another objective of this study was to verify if swimming can change oxidative stress in neuronal and skeletal muscle tissue. Studies show that oxidative stress is present in the pathophysiological process of DMD, because there is an imbalance between the formation of oxidizing agents and the antioxidant activity [21-23]. Oxidative stress is present in DMD, in skeletal muscle, and also in the CNS [21]. One of the effects of exercise is the increase in the antioxidant activity. The exercise produces ORS, which act as signals of molecular events, which regulate muscle cell adaptations, such as the regulation of antioxidant enzymes [24]. In this study, it was observed that swimming increased free thiols, demonstrating that physical exercise was able to increase glutathione antioxidant levels, in agreement with previous studies that demonstrated the protective role of the 
exercise $[25,26]$. Free thiols are an indirect measure of the glutathione activity, an antioxidant that is present in greater numbers in the CNS [27].

In addition to verifying the influence of swimming on encephalic impairment, the evaluation of some skeletal muscle tissues is necessary. As DMD is an essentially neuromuscular disease, it is necessary to include skeletal muscle assessments (gastrocnemius and diaphragm), since the main characteristic of this disease is related to the involvement of these structures with calf pseudohypertrophy, frequent falls, gait loss, and cardiorespiratory dysfunctions [25]. Evidence suggests that oxidative stress is associated with the aggravation of both respiratory and muscular pathology in these patients [19]. In addition, there are studies that show that exercise can protect against oxidative stress in skeletal muscle in $m d x$ mice [4, 13]. The protocol used in this study protected against the increase in lipid peroxidation in gastrocnemius in $m d x$ animals. Swimming for four weeks was able to prevent the increase in protein carbonylation observed in the gastrocnemius muscle of $m d x$ animals. This finding is in line with a study conducted in 2015 which used a swimming protocol and found a decrease in protein carbonylation [11]. The same occurred with the diaphragm muscle, where the $m d x$ animals showed significantly higher lipid peroxidation levels when compared to the non-exercised $m d x$ animals, demonstrating that the exercise protocol for four weeks was able to prevent the increase in lipid peroxidation. However, swimming was not able to reverse the increase in protein carbonation in diaphragm [4]. Considering what was exposed, it can be suggested that moderate intensity aerobic exercise - swimming - can reduce oxidative stress by increasing antioxidant defenses such as the glutathione, considering the increase in the formation of free thiols shown in the results of this study. However, as there is still no consensus in the literature on volume, frequency, and intensity of exercise in the treatment of $D M D$, this study can help to propose new perspectives for the therapeutic use of the exercise in this disease treatment.

\section{Conclusions}

Based on the lactate measurement classification, the swimming protocol used in this study was considered of moderate intensity. This protocol, applied to $m d x$ mice, was able to prevent memory impairment and oxidative stress in gastrocnemius and in most of the analyzed structures of the CNS, with significant increase in the antioxidant activity.

\section{Declarations}

\section{Acknowledgments}

This research was supported by grants from Unisul (CMC).

\section{Availability of Data}

Data will be made available upon request.

\section{Author Contributions}


Priscila Mantovani Nocetti, Viviane Freiberger and Letícia Ventura planned the experiments and wrote the paper. Clarissa Martinelli Comim managed the project and performed most of the experiments and analysis and provided input on statistical analysis and interpretation. Adriano Alberti provided substantial input for the manuscript and wrote the paper. Daniel Fernandes Martins, Leoberto Ricardo Grigollo, Cristina Salar Andreau, Rudy José Nodari Junior provided technical guidance and supervision. All authors read and approved the final manuscript.

\section{Funding}

This work was supported by a grant from the UNIEDU/FUMDES

\section{Compliance with Ethical Standards Conflict of Interest}

The authors declare that they have no conflict of interest.

\section{Consent to Participate}

Not applicable

\section{Consent for Publication}

Not applicable

\section{References}

1. Sinha R, Sarkar S, Khaitan T, Dutta S. Duchenne muscular dystrophy: Case report and review. J Fam Med Prim Care 2017;6(3):654.

2. Hoepers A, Alberti A, Freiberger V, Ventura L, Grigollo LR, Andreu CS, et al. Effect of Aerobic Physical Exercise in an Animal Model of Duchenne Muscular Dystrophy. J Mol Neurosci 2020;70:1-13.

3. Yucel N, Chang AC, Day JW, Rosenthal N, Blau HM. Humanizing the mdx mouse model of DMD: the long and the short of it. npj Regen Med. 2018;3(1):1-11.

4. Gevaerd MS, Domenech SC, Junior NGB, Higa DF, Lima-Silva AE. Alterações fisiológicas e metabólicas em indivíduo com distrofia muscular de Duchenne durante tratamento fisioterapêutico: um estudo de caso. Fisiot Mov 2020;23:93-103.

5. Le Rumeur E. Dystrophin and the two related genetic diseases, Duchenne and Becker muscular dystrophies. Bosn J Basic Med Sci 2015;15:14-20.

6. Alemdaroğlu $\bigoplus$, Karaduman A, Yilmaz ÖT, Topaloğlu H. Different types of upper extremity exercise training in duchenne muscular dystrophy: effects on functional performance, strength, endurance, and ambulation. Muscle \& Nerve 2015;51:697-705.

7. Hyzewicz J, Ruegg UT, Takeda S. Comparison of experimental protocols of physical exercise for mdx mice and duchenne muscular dystrophy patients. J Neuromuscul Dis 2015;2:325-42. 
8. Jansen M, Alfen NV, Geurts ACH, Groot IJM. Assisted bicycle training delays functional deterioration in boys with duchenne muscular dystrophy: the randomized controlled trial "No Use Is Disuse". Neurorehabilit Neural Repair 2013;27:816-27.

9. Nikolai AL, Novotny BA, Bohnen CL, Schleis KM, Dalleck LC. Cardiovascular and Metabolic Responses to Water Aerobics Exercise in Middle-Aged and Older Adults. J Phys Act Health 2009;6:333-8.

10. Hyzewicz J, Tanihata J, Kuraoka M, Ito N, Miyagoe-Suzuki Y, Takeda S. Low intensity training of mdx mice reduces carbonylation and increases expression levels of proteins involved in energy metabolism and muscle contraction. Free Radic Biol Med 2015;82:122-36.

11. Mazzardo-Martins L, Martins DF, Marcon R, Santos UD, Speckhann B, Gadotti VM et al. High-intensity extended swimming exercise reduces pain-related behavior in mice: involvement of endogenous opioids and the serotonergic system. J. Pain 2010;11:1384-93.

12. Ishii $\mathrm{H}$, Nishida Y. Effect of lactate accumulation during exercise-induced muscle fatigue on the sensorimotor cortex. J Phys Ther Sci 2013;25:1637-42.

13. Leussis MP, Bolivar VJ. Habituation in rodents: A review of behavior, neurobiology, and genetics. Neurosc and Biobehav Rev 2006;30:1045-64.

14. Ogihara CA, Schoorlemmer GH, Lazari MF, Giannocco G, Lopes OU, Colombari E, Sato MA. Swimming Exercise Changes Hemodynamic Responses Evoked by Blockade of Excitatory Amino Receptors in the Rostral Ventrolateral Medulla in Spontaneously Hypertensive Rats. BioMed Res Int 2014;1-10.

15. Levine A, Tenhaken R, Dixon R, Lamb C. H2O2 from the oxidative burst orchestrates the plant hypersensitive disease resistance response. Cell 1994;79:583-93.

16. Schubert MM, Washburn RA, Honas JJ, Lee J, Donnelly JE. Exercise volume and aerobic fitness in young adults: the Midwest Exercise Trial-2. Springerplus 2015;5:183.

17. Gaesser GA, Poole DC. The slow component of oxygen uptake kinetics in humans. Exerc Sport Sci Rev 1996;24:35-71.

18. Comim CM, Tuon L, Stertz L, Vainzof M, Kapczinski F, Quevedo J (2009) Striatum brain-derived neurotrophic factor levels are decreased in dystrophin-deficient mice. Neurosci Lett 2009;459:66-8.

19. Araujo GG, Papoti M, Reis IG, Mello MA, Gobatto CA. Physiological responses during linear periodized training in rats. Eur J Appl Physiol 2012;112:839-52.

20. Cechella JL, Leite MR, Gai RM, Zeni G. The impact of a diphenyl diselenide-supplemented diet and aerobic exercise on memory of middle-aged rats. Physiol Behav 2014;135:125-9.

21. Lawler JM. Exacerbation of pathology by oxidative stress in respiratory and locomotor muscles with Duchenne muscular dystrophy. J Physiol 2011;589:2161-70.

22. Moraes LH, Bollineli RC, Mizobuti DS, Silveira L dos R, Marques MJ, Minatel E. (2015) Effect of Nacetylcysteine plus deferoxamine on oxidative stress and inflammation in dystrophic muscle cells. Redox Rep 2015;20:109-5. 
23. Kozakowska M, Pietraszek-Gremplewicz K, Jozkowicz A, Dulak J. The role of oxidative stress in skeletal muscle injury and regeneration: focus on antioxidant enzymes. J Muscle Res Cell Motil 2015;36:377-93.

24. Gomez-Cabrera MC, Domenech E, Viña J. Moderate exercise is an antioxidant: Upregulation of antioxidant genes by training. Free Radic Biol Med 2008;44:126-31.

25. Antoncic-Svetina M,Sentija D, Cipak A, Milicic D, Meinitzer A, Tatzber F. Ergometry induces systemic oxidative stress in healthy human subjects. Tohoku J Exp Med 2010;221:43-8.

26. Jia B, Wang X, Kang A, Wang X, Wen Z, Yao W et al. The effects of long term aerobic exercise. Clin. Hemorheol Microcirc 2012;51:117-27.

27. Halliwell B (1992) Reactive oxygen species and the central nervous system. J Neurochem 1992;59:609-23.

\section{Figures}

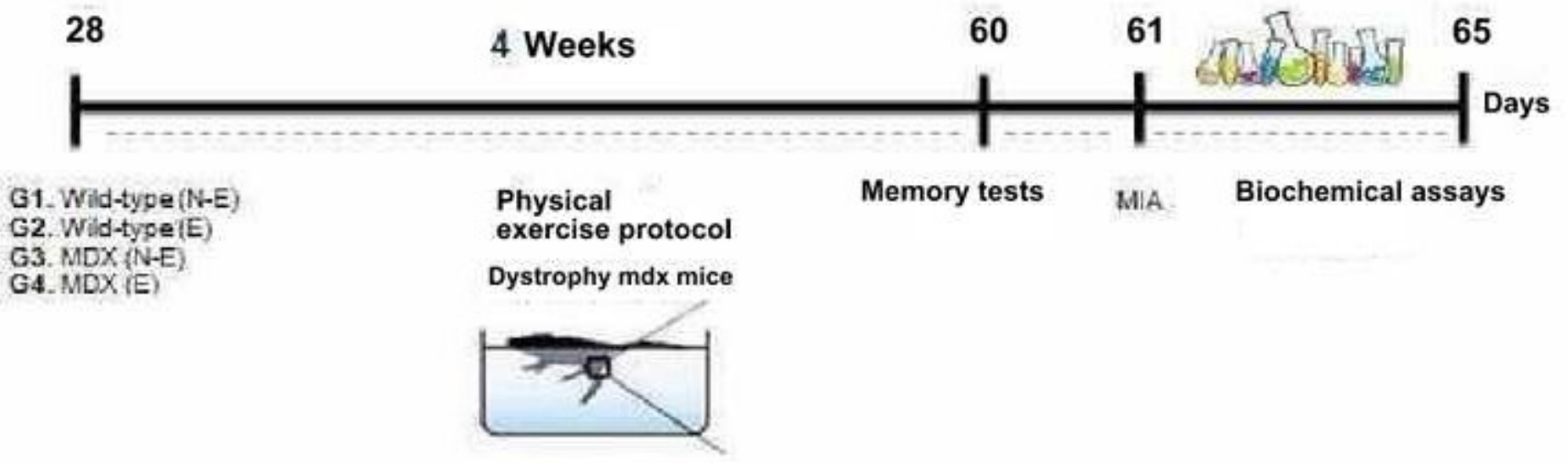

\section{Figure 1}

As illustrated in Fig. 1. 


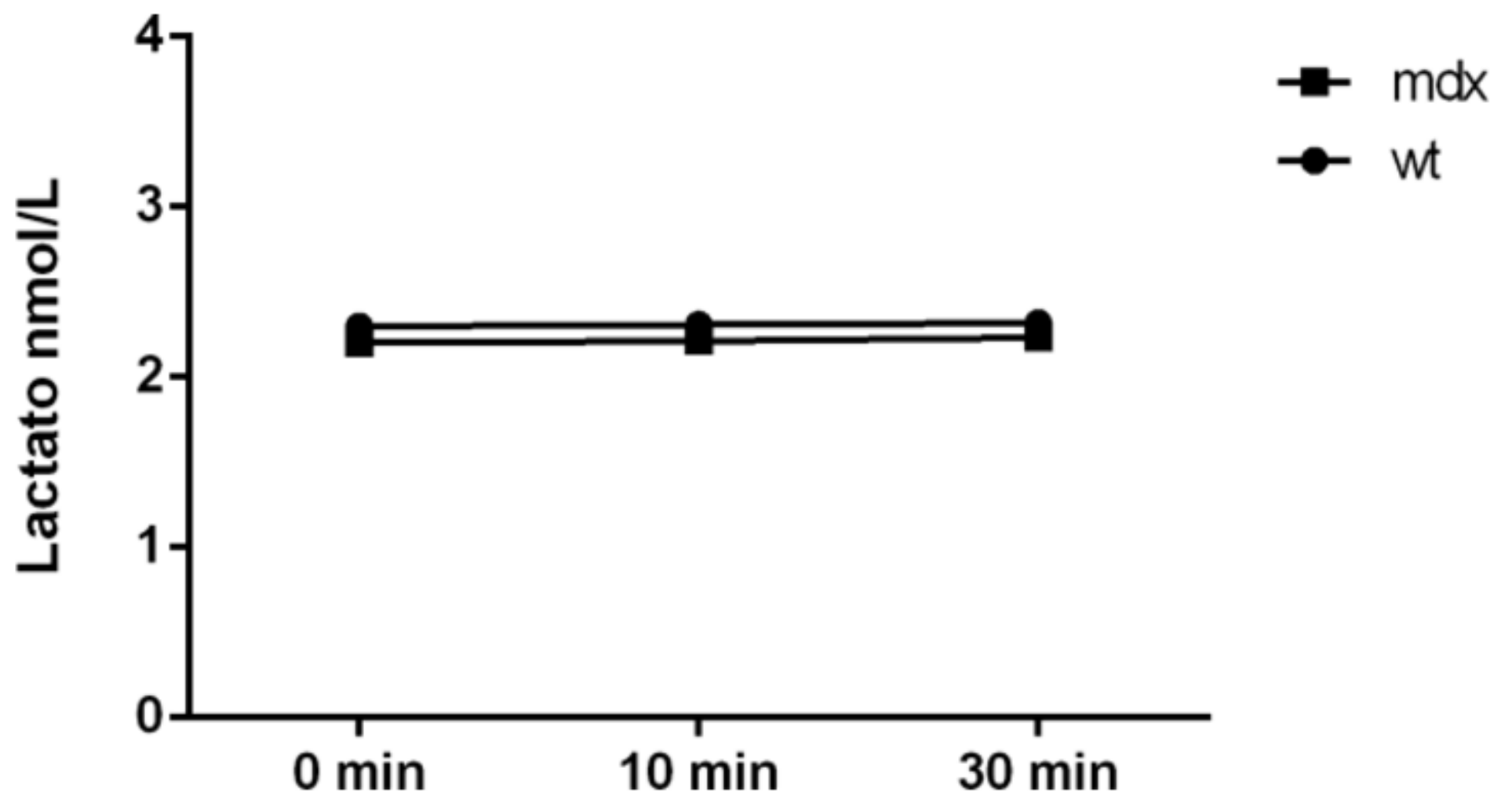

Figure 2

The results obtained after the lactate measurement made in the blood taken from the animals during the performance of the swimming protocol. 
A)

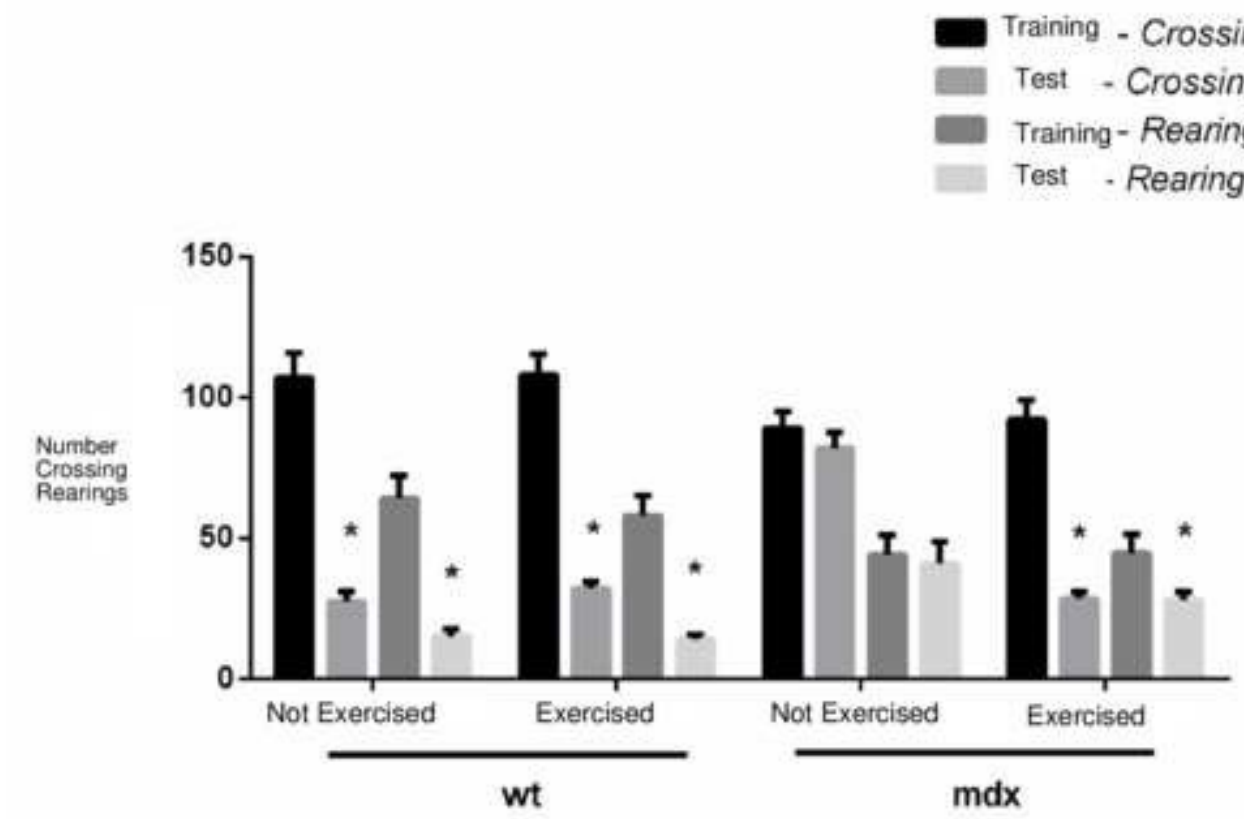

B)

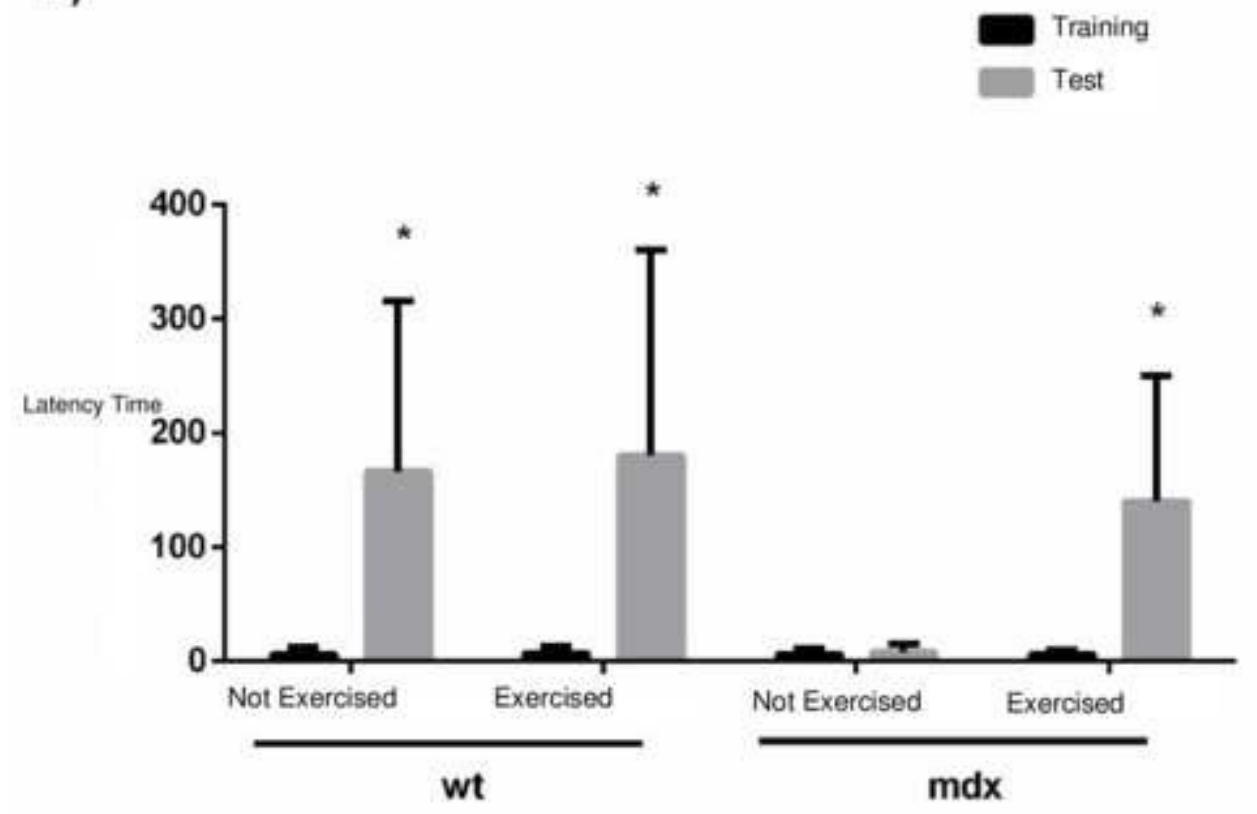

Figure 3

The results obtained after the application of the swimming protocol on aversive memory (Fig. 3A) and habituation (Fig. 3B). In Fig. 3A, the results of the habituation memory evaluation, through the open field test, can be observed. 
A)

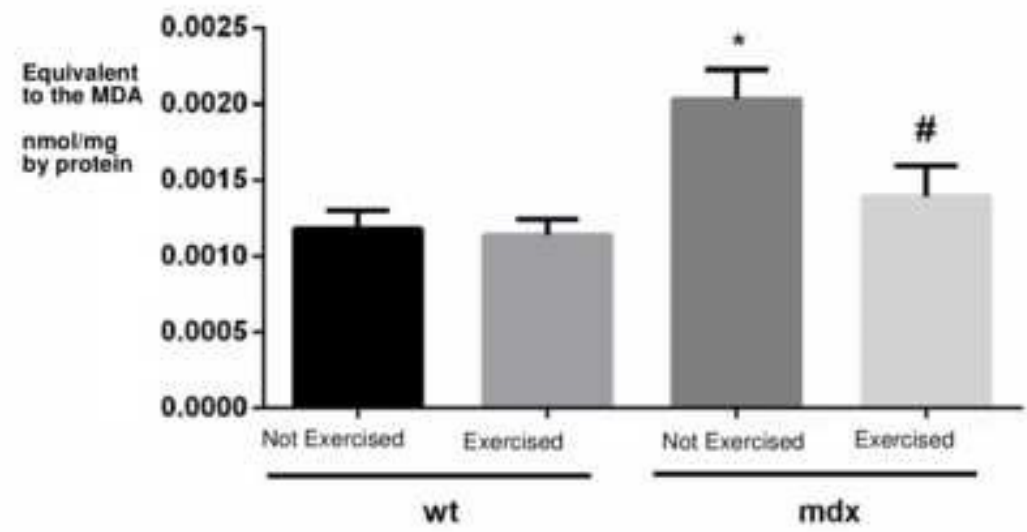

B)

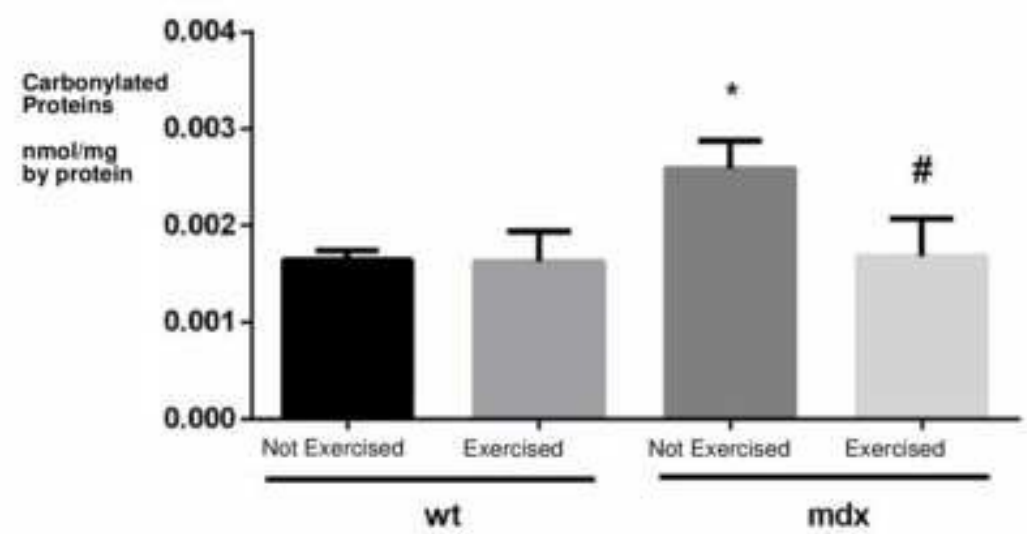

C)

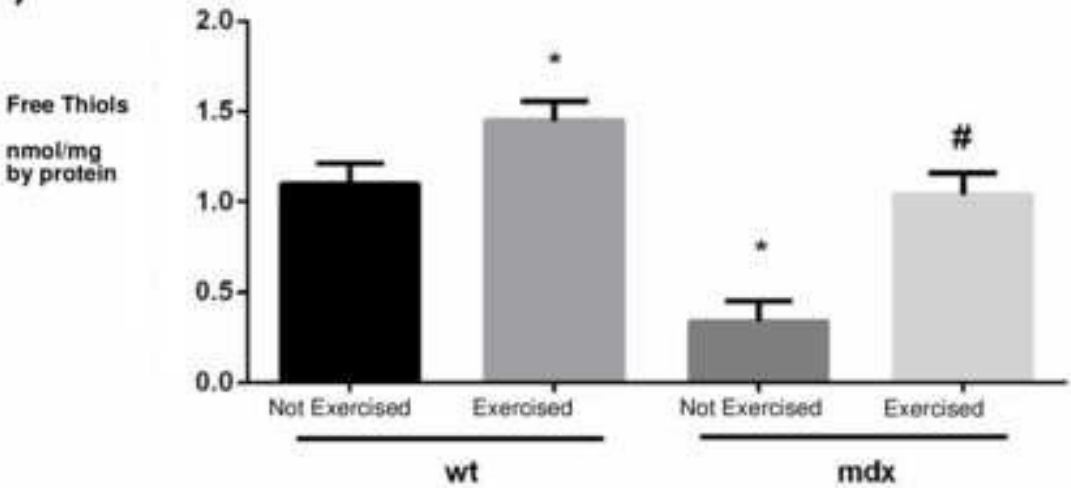

Figure 4

The results of using a swimming protocol on lipid peroxidation (Fig. 4A), protein carbonylation (Fig. 4B), and free thiols (Fig. 4C) in the gastrocnemius muscle. 
A)

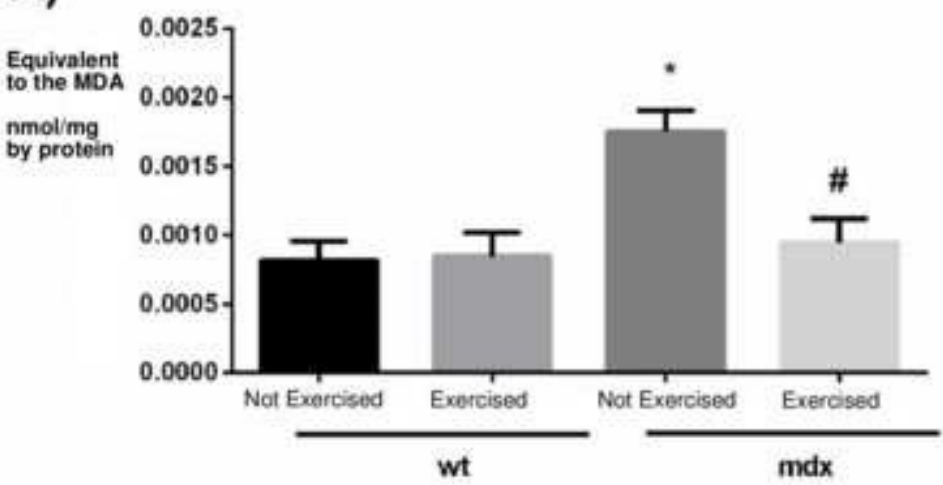

B)

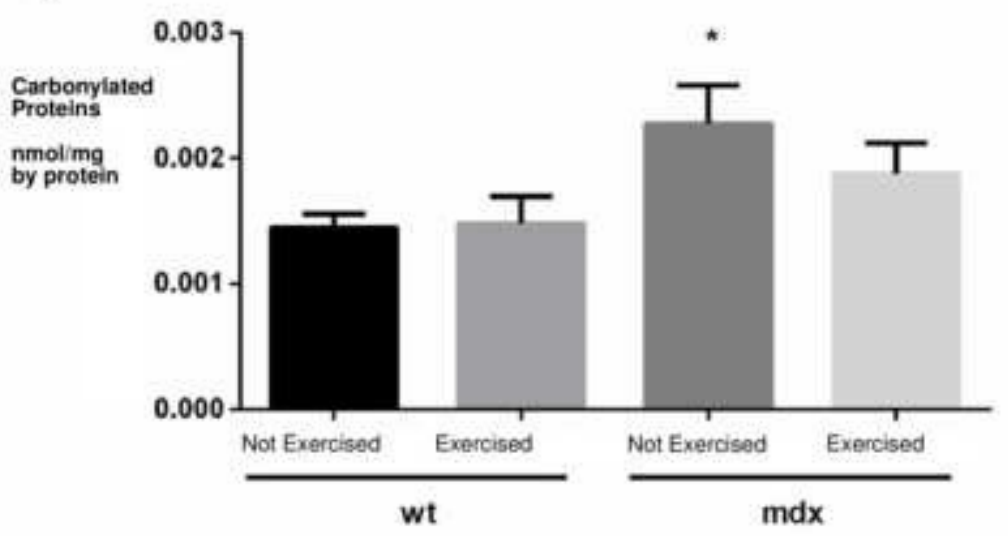

C)

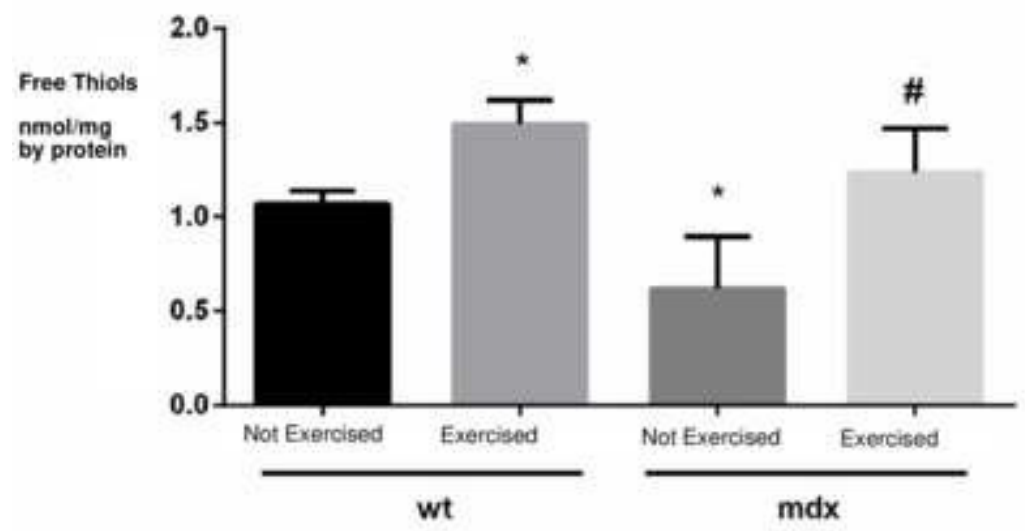

Figure 5

The results of using a swimming protocol on lipid peroxidation (Fig. 5A), protein carbonylation (Fig. 5B), and free thiols (Fig. 5C) in the diaphragm muscle. 
A)

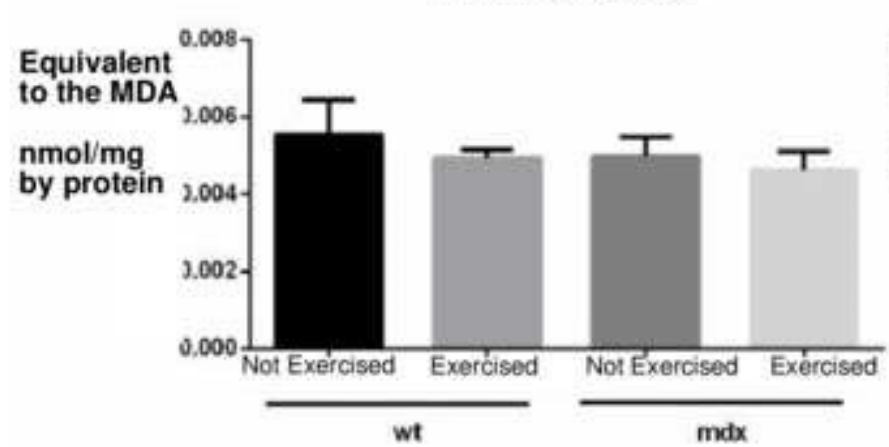

C)

Striatum

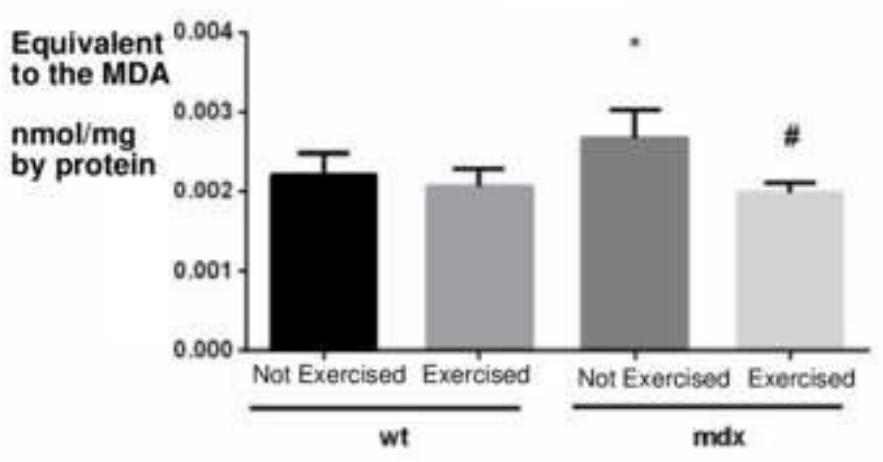

B)

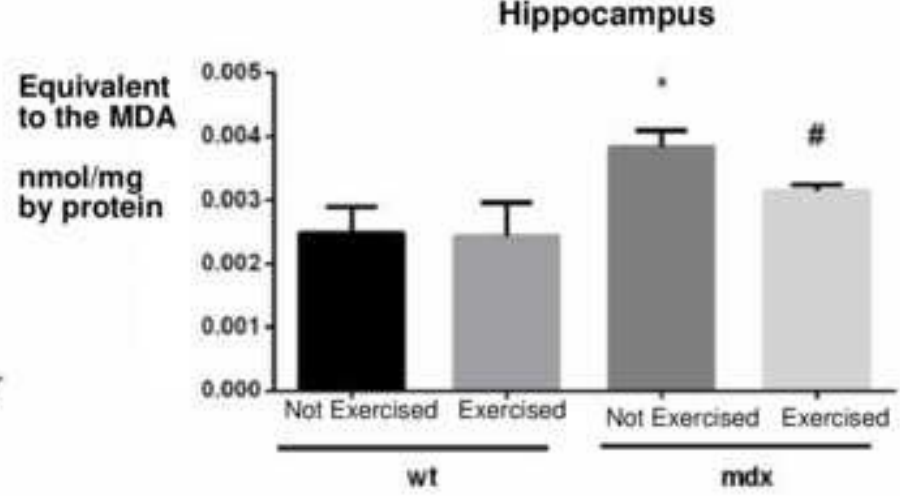

D)

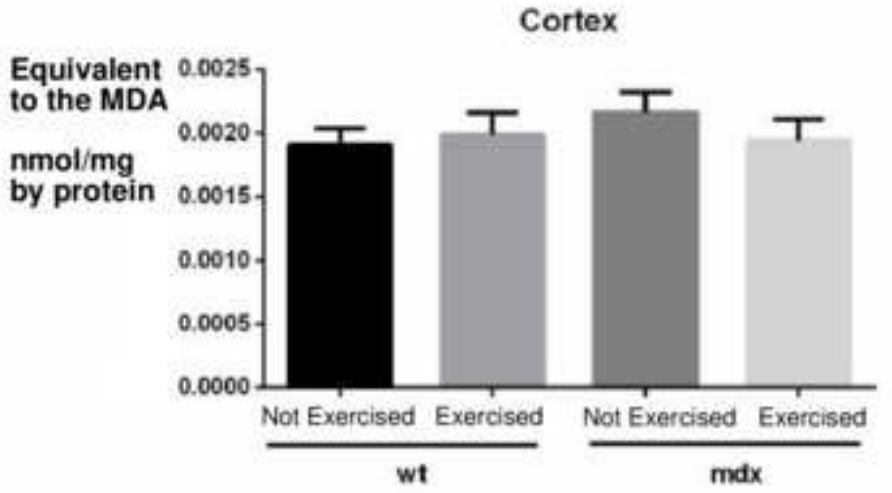

\section{Figure 6}

The results of using a swimming protocol on lipid peroxidation in the prefrontal cortex (Fig. 6A), hippocampus (Fig. 6B), striatum (Fig. 6C), and cortex (Fig. 6D). 
A)

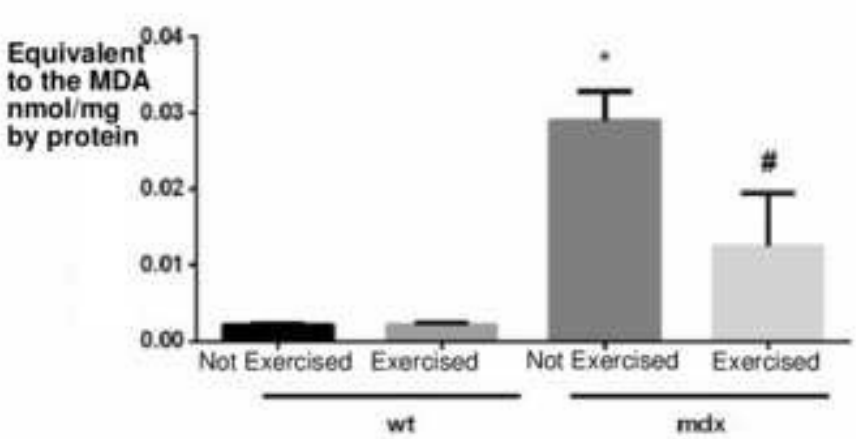

C)

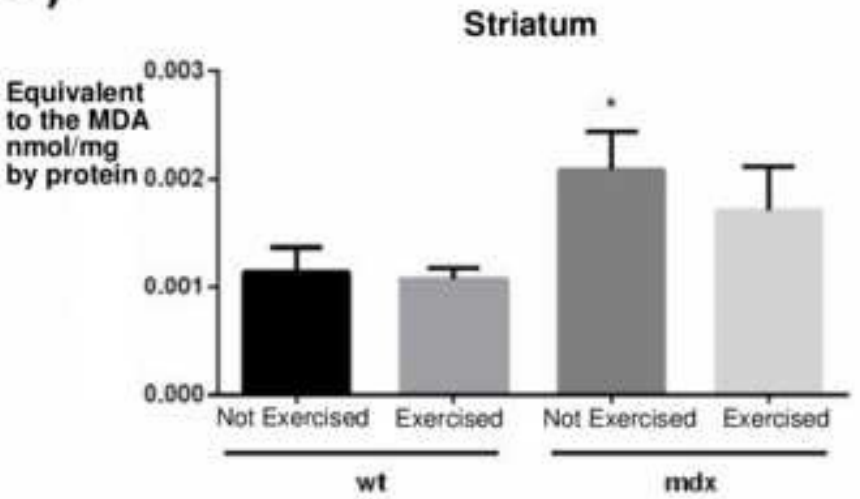

\section{B)}

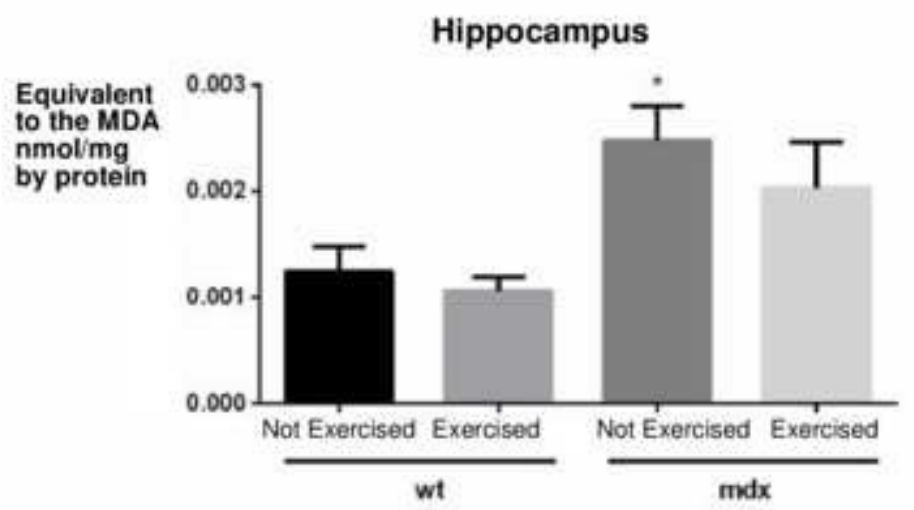

\section{D)}

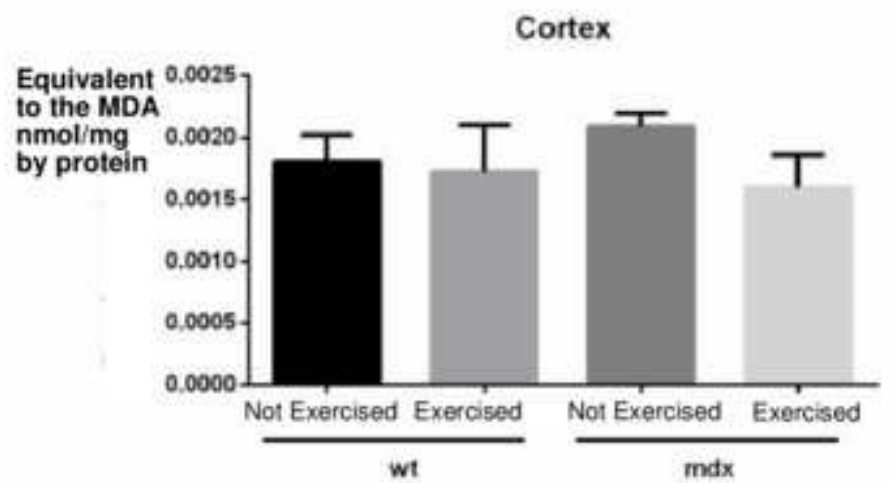

\section{Figure 7}

The results of using a swimming protocol on protein carbonylation in the prefrontal cortex (Fig. 7A), hippocampus (Fig. 7B), striatum (Fig. 7C) and cortex (Fig. 7D). 
A)

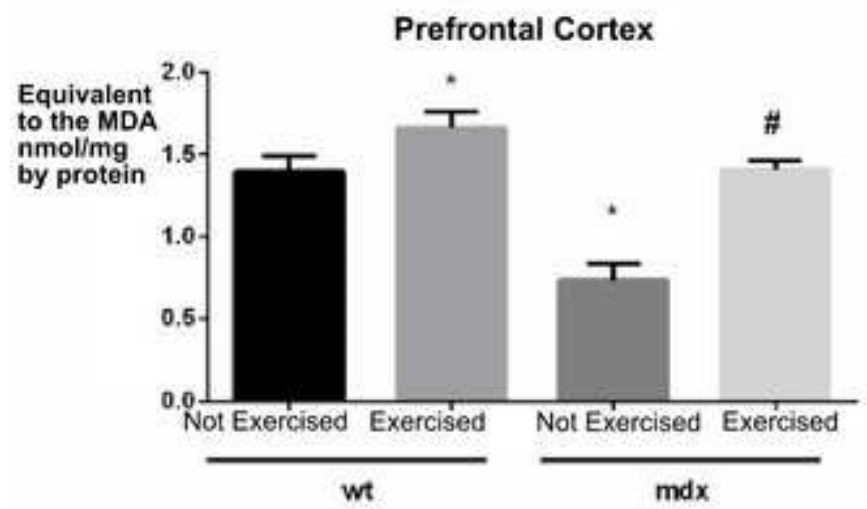

C)

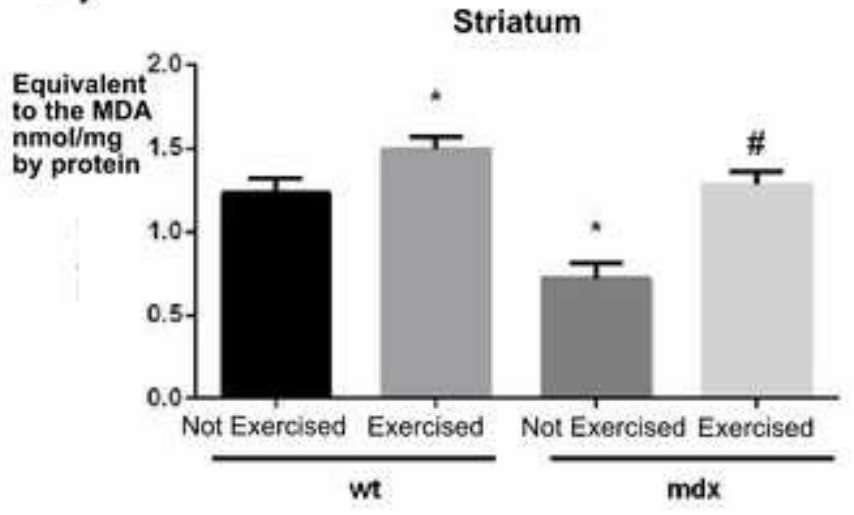

B)

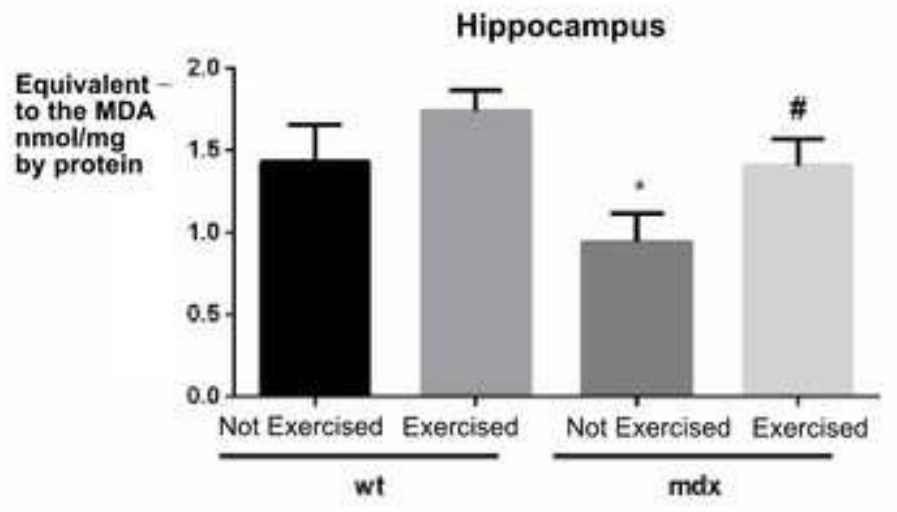

D)

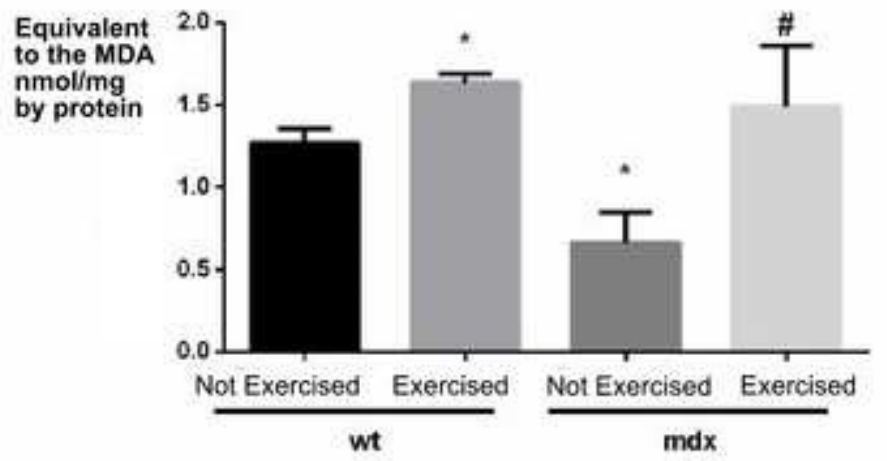

\section{Figure 8}

The results of using a swimming protocol on free thiols in the prefrontal cortex (Fig. 8A), hippocampus (Fig. 8B), striatum (Fig. 8C) and cortex (Fig. 8D). 